Therapienaive HIV-Patienten

\title{
Fortbildungsinitiative für frühen Behandlungsbeginn
}

- Im Rahmen der Münchner Aidstage wurde die internationale Fortbildungsinitiative BEST (Better equipped for Starting Treatment) vorgestellt. Ziel ist, therapienaive HIV-Patienten über den optimalen Behandlungsstart zu informieren und so Erkrankung und antiretrovirale Therapie mit dem Alltag der Betroffenen in Einklang zu bringen. Die Fortbildungsveranstaltungen werden von Bristol-Myers Squibb und Gilead Sciences unterstützt. Sie erfolgen in Form von Workshops „von Betroffenen für Betroffene".

Beim ersten Workshop berichteten Dr. Ulrich Seybold und Engelbert Zankl von der Münchner Aidshilfe e.V. über das Projekt. Bei der schwierigen Entscheidung über den Beginn der antiretroviralen Therapie werden objektive Informationen zum Infektionsgeschehen und zu den Behandlungsempfehlungen gebraucht. „Es gilt, ein realistisches Bild für die persönliche Erkrankungssituation zu entwickeln, damit HIV-infizierte Menschen, zusammen mit ihrem Arzt, die individuell richtige Entscheidung treffen können“, so Seybold.

Nach aktuellen klinischen Daten bietet ein früher Therapiestart prognostische Vorteile. Nationale und internationale Leitlinien empfehlen, $a b$ einer CD4-Zell$\mathrm{zahl}<350 / \mu \mathrm{l}$ mit der Behandlung zu beginnen. Gelingt es, durch die antiretrovi- rale Therapie die Viruslast unter die Nachweisgrenze zu bringen, steigt die Zahl der CD4-Zellen allmählich wieder an und damit die Immunfunktion des Patienten.

- Jürgen Setton

Quelle: Symposium, Münchner AIDS-Tage,

6. März 2010 (Veranstalter: Bristol-Myers

Squibb und Gilead Sciences)

\section{Compliance erleichtern, Resistenzrisiko senken}

\section{HAART mit einmal täglich einer Tablette}

Die fixe Kombination aus Tenofovir, Emtricitabin und Efavirenz (Atripla ${ }^{\circledR}$ ) bringt eine deutliche Erleichterung für HIV-Patienten. Mit diesem Präparat kann ein vollständiges HAART-Regime mit einmal täglich einer Tablette eingenommen werden. Das erhöht die Chance, dass die Therapie regelmäßig genommen wird und sich keine Resistenzen entwickeln. Die Kombination ist zugelassen für Patienten, bei denen HIV unter einer initialen Therapie seit mindestens drei Monaten nicht mehr nachweisbar ist.

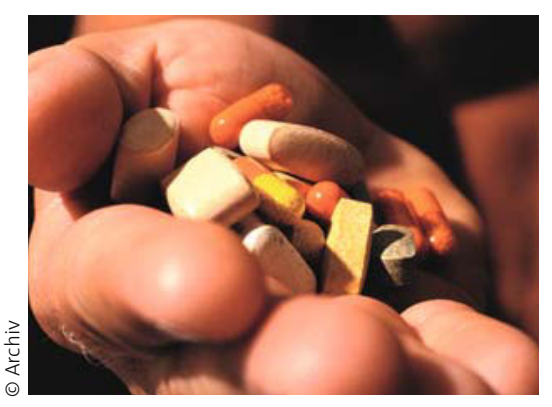

So viele Tabetten muss man HIVPatienten heute nicht mehr zumuten.

\section{Umdenken in der Schmerztherapie Opioide nicht erst als Ultima Ratio}

— Die Therapie von Schmerzen sollte sich nicht an einem starren Stufenschema orientieren, sondern die Entstehungsmechanismen berücksichtigen. Deshalb kann eine frühzeitige Behandlung mit Opioiden sinnvoll sein.

Wie Dr. Gerhard Müller-Schwefe, Göppingen, betonte, sollten entzündungshemmende Substanzen der WHO-Stufe I nur dann zum Einsatz kommen, wenn eine Entzündung die Ursache für Schmerzen ist. Dem Schmerzexperten zufolge hat sich gerade bei älteren Menschen mit degenerativen Erkrankungen der Gelenke Buprenorphin als 7-Tage-Pflaster (Norspan $^{\circledast}$ ) in der Erstlinientherapie bewährt. Buprenorphin biete besonders für ältere, multimorbide Patienten Vorteile wie die unabhängig von der Nierenfunktion kalkulierbare Wirksamkeit und die gute
Kombinierbarkeit mit anderen Substanzen.

Bei einer Opioidvortherapie empfiehlt es sich, ein Pflaster mit ausreichend hoher Startdosis zu wählen. Bei Patienten, die bisher mit Tramadol oder Tilidin 200 mg/ $d$ behandelt wurde, ist in der Regel ein Einstieg mit $10 \mu \mathrm{g} / \mathrm{h}$ des 7 -Tage-Pflasters sinnvoll. „Ist die Dosis nicht hoch genug für eine effektive Analgesie, lassen sich das beim Patienten verlorene Vertrauen und die Compliance nur schwer wiederherstellen“, betonte Ulf Schutter, Marl.

\section{Auch gegen neuropathische} Schmerzkomponenten wirksam Dass eine Umstellung sinnvoll sein kann, belegen die Daten einer bisher nicht veröffentlichten randomisierten Doppelblindstudie, so Schutter. Darin konnte für das 7-Tage-Pflaster eine deutliche Überlegenheit gegenüber der Vortherapie gezeigt werden, wobei $50 \%$ der Patienten mit chronischen Rückenschmerzen (Mixed Pain) mit einem anderen Opioid vorbehandelt waren.

Buprenorphin hat eine ausgeprägte analgetische Wirksamkeit am $\mu$-Rezeptor und eine direkte Wirkung an spannungsabhängigen Natriumkanälen, erläuterte Prof. Wolfgang Koppert, Hannover. Dies macht das Opioid auch für die Behandlung neuropathischer Schmerzen geeignet. Weiterhin dürfte dieser Effekt auch an der antihyperanalgetischen Wirkung von Buprenorphin beteiligt sein.

\footnotetext{
- Ralf Hausmann

Quelle: Pressegespräch: „Differenzierte Therapie mit Opioiden“, Frankfurt/M., 19. März 2010 (Veranstalter: Grünenthal)
} 